\title{
Mejora de los resultados académicos usando lecciones interactivas en $\mathrm{R}$ Improving academic achievement using interactive lessons in $\mathrm{R}$
}

\author{
Isabel Parra-Frutos \\ ipf@um.es \\ Departamento de Métodos Cuantitativos para la Economía y la Empresa \\ Universidad de Murcia \\ Murcia, Spain
}

\begin{abstract}
Resumen- Se han diseñado unas lecciones interactivas con el paquete swirl de $\mathrm{R}$ que hacen un recorrido por el temario de una asignatura de Estadística. Estas lecciones incluyen explicaciones que pueden apoyarse en esquemas o gráficos junto con ejemplos realizados por los estudiantes para reforzar las explicaciones y problemas para practicar. El contenido se desarrolla de forma secuencial, sin posibilidad de saltar ninguna pregunta, y con penalización en las respuestas incorrectas que deben ser corregidas por el propio estudiante para poder continuar. Las lecciones han sido un trabajo extra para los estudiantes, pero ha sido bonificado para incentivar su realización y con una pauta temporal. Los resultados obtenidos están en sintonía con el consenso generalizado en la literatura que apoya la mejora de los resultados académicos con el uso de actividades mejoradas por la tecnología.
\end{abstract}

Palabras clave: Aprendizaje mejorado por la tecnología (TEL), R, lecciones interactivas

Abstract- Some interactive lessons have been made using the swirl package for $\mathrm{R}$ for explaining the content of a subject of Statistics. These lessons include explanations using schemes or graphics, examples to be solved by students for a better understanding and exercises to practice. The lessons are developed sequentially, without possibility of skipping questions and with penalties for wrong answers that should be corrected by students to continue. These activities have been an extra homework for students, with timing and incentives for completing them. The present study's finding supports the apparent consensus of the literature about the improvement of academic achievement when using activities based on technology.

Keywords: Technology enhanced Learning (TEL), R, interactive lessons

\section{INTRODUCCIÓN}

Desde hace unos años las actividades online se están utilizando cada vez más por los docentes por el ahorro de tiempo a la hora de ponerlas y corregirlas (Angus \& Watson, 2009). A su vez, los estudiantes obtienen un doble beneficio, por un lado, un feedback instantáneo $\mathrm{y}$, por otro, una flexibilidad en cuanto al momento y lugar donde realizarlas. A su vez, los estudiantes tienen una visión positiva de su uso (Kennedy \& Dunn, 2018) y esperan verlas como parte de sus estudios (Margaryan, Littlejohn, \& Vojt, 2011).

De acuerdo con Dunn and Kennedy (2019), este tipo de material es una forma de e-learning y formaría parte de lo que se denomina aprendizaje mejorado mediante el uso de tecnología (Technology Enhanced Learning, TEL). El software educativo puede tener beneficios pedagógicos (Beetham and Sharpe 2013) y dar resultados académicos positivos (Fonseca et al. 2014). Sin embargo, el uso de la tecnología no parece suficiente para mejorar la educación. Lin, Liu, and Yuan (2001) obtienen que cuando se realizan actividades online los estudiantes aprenden si están altamente motivados. Las motivaciones pueden ser intrínsecas (el proceso de aprendizaje es percibido como un beneficio en sí mismo) o extrínsecas (el deseo de obtener una calificación alta, obtención de una recompensa externa). Ambas motivaciones ya fueron asociadas en general con el éxito académico por Whitehead (1984). De acuerdo con Dunn and Kennedy (2019), ambas motivaciones conducen a que el estudiante se involucre con actividades TEL y cuanto mayor es este compromiso o implicación mayor es la calificación obtenida. Sin embargo, se ha observado un nivel de uso por parte del estudiante menor del esperado dentro de la educación superior en Reino Unido (UCISA, 2016). Por otro lado, Huang et al. (2017) muestran que la implementación de una técnica específica de aprendizaje basado en TEL mejora el rendimiento académico, pero no la motivación para aprender. En este sentido, el compromiso con la educación y el éxito académico requiere no solo un comportamiento (asistir a clase, hacer las actividades) sino un componente cognitivo (el estudiante siente un reto) y un componente emocional (emociones positivas hacia la asignatura), ambos considerados motivaciones intrínsecas del estudiante (Kahu 2013, Fredricks, Blumenfeld y Paris 2004, y Wang y Eccles 2012). A nivel de actividades TEL, las motivaciones intrínsecas predicen que el estudiante se involucre con ellas, mientras que las motivaciones extrínsecas predicen su uso, sin embargo, solo el compromiso (con sus tres componentes) puede predecir la calificación (Dunn and Kennedy, 2019).

Parece haber un amplio consenso general en la literatura que apoya la mejora de los resultados académicos con el uso de herramientas online (en general, TEL). Angus and Watson (2009) encuentran que una mayor exposición a los instrumentos online conduce a un mayor aprendizaje del alumno. Kibble (2007) muestra una correlación significativa entre la nota de los tests online y el examen final. McSweeney and Weiss (2003) revelan que los estudiantes que usaron una herramienta matemática online (the "Math Online" Tool) obtuvieron notas significativamente mayores que los que no. Henly (2003) afirma que para el $92 \%$ de los estudiantes la evaluación online a través de tests o preguntas cortas les ayudaba en su aprendizaje. 


\section{CONTEXTO}

Con el objetivo de mejorar los resultados obtenidos en Estadística por los estudiantes de Ciencias Sociales, hemos desarrollado una serie de lecciones interactivas con el paquete swirl de R. En particular, han estado disponibles para los estudiantes de una asignatura de Estadística Descriptiva del primer cuatrimestre de primero del Grado en Economía de la Universidad de Murcia en el curso 2018-2019. Estas lecciones permiten exponer de forma secuencial y guiada tanto problemas como conceptos con ejemplos de aplicación. Se trata de hacer trabajar más al estudiante para que aprenda más de una forma dirigida sin que suponga una carga extra para el docente. Desde nuestro punto de vista, para adquirir habilidades estadísticas o matemáticas es necesario practicarlas y estas lecciones dan esa oportunidad al estudiante. Trataremos de analizar el nivel de implicación de los estudiantes en este tipo de actividades y si este material en particular mejora los resultados académicos.

\section{DESCRIPCIÓN}

En su origen las lecciones swirl han sido diseñadas para aprender R, el lema en su página web (https://swirlstats.com/) es “Learn R, in R". La web también proporciona un enlace a un repositorio de lecciones swirl de aprendizaje de R. Sin embargo, este tipo de lecciones se pueden usar también para el aprendizaje de cualquier materia (cuantitativa $o$ no cuantitativa). Nosotros las utilizamos con el objetivo de mejorar el aprendizaje y comprensión de la Estadística Descriptiva sin entrar en el análisis de datos con R, que sería diferente.

Las lecciones swirl pueden proporcionar beneficios en cualquier disciplina, no solo en las cuantitativas, dada la variedad de preguntas que permite usar. En el caso de materias cuantitativas se puede, además, combinar problemas de cálculo con cualquier otro tipo de preguntas para comprobar que el estudiante ha asimilado lo explicado o para, simplemente, practicarlo. Estas lecciones permiten intercalar texto, imágenes (con fórmulas, gráficos, esquemas, ...) y preguntas de una forma sucesiva y pausada. Se pueden incluir cuestiones tipo test o con respuesta en forma de operación matemática, en forma numérica y de texto. También pueden abrir webs o videos de Internet en el navegador. Los datos de los ejemplos o ejercicios pueden ser aleatorios, de esta forma se consigue que cada estudiante tenga ejercicios diferentes al del resto de compañeros o que cada vez que realice la lección tenga datos nuevos.

El estudiante debe ir respondiendo (en la consola de RStudio) a las preguntas que se van planteando, de tal forma que, hasta que no responda correctamente, la lección no puede avanzar. Esta metodología permite poner en práctica y de forma inmediata las explicaciones que preceden a la pregunta que tiene que responder siendo, además, el feedback inmediato. Así, mediante las lecciones swirl se puede exponer un tema concreto de forma secuencial (no es posible saltarse ninguna parte) y guiado. También se pueden programar para que un estudiante pueda ir saltando aquellas preguntas que no sabe responder, pero en nuestro caso hemos deshabilitado esta opción.

Todas las cuestiones pueden incluir un texto de ayuda que se muestra cuando el estudiante no proporciona la respuesta correcta. Este texto puede ser simplemente una indicación de que la respuesta es incorrecta, una pista para la resolución o directamente la respuesta correcta que el alumno puede copiar y pegar. Otras características de estas lecciones son: informan continuamente del porcentaje de lección completada, permiten salir temporalmente para hacer cálculos y luego volver a la pregunta donde se dejó, y guarda el progreso de una lección si se abandona antes de terminarla.

El estudiante no necesita conocer $\mathrm{R}$ ni RStudio para la realización de estas lecciones, sin embargo, su realización le va introduciendo en el uso de este software. Desde el punto de vista del docente son fáciles de diseñar, aunque pueden ser tan complejas como se quiera dependiendo del nivel de conocimientos de R. En el diseño de la respuesta correcta es posible utilizar toda la versatilidad y funcionalidad de $\mathrm{R}$, así como en el planteamiento de problemas a través de RScripts.

El docente puede controlar la realización de una lección por parte del estudiante dado que esta proporciona un código asociado al DNI que se introduce al finalizar. La elaboración del código se debe modificar cada curso académico para un mayor control. Las lecciones diseñadas también calculan una nota que depende del total de intentos fallidos acumulados. Una vez realizada la lección, esta proporciona un certificado, descargable en formato pdf, diseñado por el docente y donde hemos establecido que aparezca el DNI, el código obtenido, la nota, el tiempo de realización y la fecha.

Hemos diseñado las lecciones para que se usen 3 de las 4 ventanas de RStudio: la consola (donde aparecen las lecciones de forma pausada), la ventana para las imágenes (esquemas, gráficos, fórmulas, etc.) y la ventana de edición (para mostrar los datos de los problemas de forma permanente).

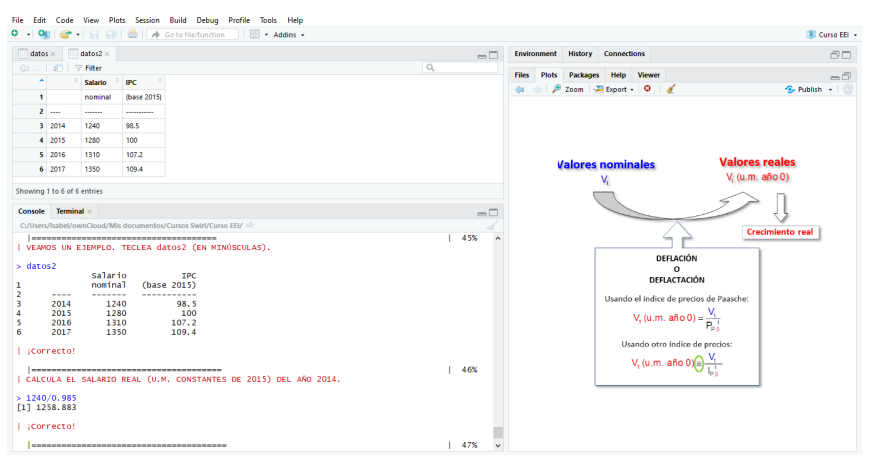

Figura 1 Lección swirl en RStudio.

Desde nuestro punto de vista, las lecciones interactivas diseñadas tienen múltiples beneficios para el estudiante: adquiere experiencia en el uso correcto de la materia explicada, permite reconducir el pensamiento lógico que desarrolla dentro de una disciplina, refuerza la comprensión mediante ejemplos realizados por el propio estudiante, fomenta un aprendizaje activo, promueve la importancia de la obtención de resultados exactos, aumenta la concentración del estudiante a la hora de estudiar la asignatura al combinarse la lectura de informaciones breves con la resolución de cuestiones, mejora la lectura comprensiva de los problemas e instrucciones para hacer alguna actividad, fomenta la atención a lo que escribe dado que el número de respuestas incorrectas cuenta e introduce al estudiante en un manejo básico del programa $\mathrm{R}$, en el caso de que no lo conozca.

A pesar de los beneficios para el estudiante, si no existe un compromiso que incluya el componente cognitivo y emocional (un deseo por aprender) las lecciones pueden ser muy frustrantes. En este sentido, no basta con hacerlas sino haber estudiado mínimamente la materia y responder con criterio o 
lógica a las preguntas. Hemos observado que, cuando el estudiante no sabe algo, tiende a probar diferentes opciones sin criterio alguno. Para evitar esto se ha incorporado una nota que penaliza los intentos fallidos. Esta nota, además, hace que el estudiante quiera volver a hacer la lección en el caso de que no haya obtenido un aprobado, lo que redunda en un mayor beneficio para el alumno y, teniendo en cuenta que los datos de los ejercicios se generan aleatoriamente, no repite exactamente los mismos ejercicios.

Las lecciones swirl se han utilizado en la asignatura de Estadística básica para la Economía cuyo temario es evaluado a lo largo del cuatrimestre mediante dos pruebas intermedias. Existen otros instrumentos de evaluación como un trabajo en grupo, un seminario y un examen final. El material disponible para los estudiantes ha sido un manual o texto-guía elaborado expresamente para la asignatura y las lecciones swirl. Las lecciones swirl se han pautado estableciendo una obligación de entregar los certificados de realización en unas fechas concretas para asegurar un adecuado ritmo de trabajo del estudiante.

Para la preparación de la primera prueba intermedia se han elaborado un total de 16 lecciones swirl, 9 de tipo teóricopráctico con explicaciones y ayudas, y otras 7 lecciones prácticas sin ayuda. Para la segunda prueba intermedia se han elaborado 7 lecciones teórico-prácticas y 7 lecciones prácticas de examen. La duración de estas lecciones para el profesor está entre 10 y 20 minutos, que se puede multiplicar por 3 o 4 para el estudiante. Su realización ha sido fundamentalmente trabajo para casa, siendo una motivación para abordarlas la obtención de un bonus en la nota de la prueba parcial correspondiente, 0.5 puntos en la prueba 1 y 0.75 en la 2 . El estudio de Kibble (2007) muestra que dar este tipo de incentivos a los estudiantes para completar las tareas online tiene como efecto un incremento importante de la participación. Sin embargo, desde nuestro punto de vista, los porcentajes de abandono de las lecciones swirl han sido altos (Tabla 1). En particular, un $45 \%$ y $68 \%$ de alumnos hacen menos de la mitad de las lecciones swirl correspondientes a la prueba 1 y 2 , respectivamente. Varios motivos pueden ser los responsables de este resultado, quizás el incentivo no fue suficiente, el tiempo que pueden requerir puede ser percibido como alto o quizás no aprecian que los beneficios de realizarlas pueden ir más allá de la bonificación directa de la nota. Como más adelante se verá, aproximadamente la mitad de los alumnos encuestados consideraron estas lecciones un trabajo excesivo. En la Tabla 1 también se puede observar que cuanto mayor es el número de lecciones realizadas, mayor es la nota media alcanzada por los estudiantes. Este resultado concuerda con el obtenido por Angus and Watson (2009).

Tabla 1 Seguimiento de las lecciones swirl.

\begin{tabular}{ccccc}
\hline & \multicolumn{2}{c}{ Prueba 1 } & \multicolumn{2}{c}{ Prueba 2 } \\
\hline $\begin{array}{c}\text { Lecciones } \\
\text { realizadas }\end{array}$ & Estudiantes & $\begin{array}{c}\text { Nota } \\
\text { media }\end{array}$ & Estudiantes & $\begin{array}{c}\text { Nota } \\
\text { media }\end{array}$ \\
\hline $0,1,2,3,4$ & $38.5 \%$ & 4.0 & $66.7 \%$ & 4.5 \\
La mitad o más & $55.0 \%$ & 5.8 & $32.3 \%$ & 4.7 \\
Todas & $28.0 \%$ & 6.8 & $24.7 \%$ & 5.3 \\
\hline
\end{tabular}

\section{Resultados}

\section{A. Opinión del estudiante}

Se ha realizado una encuesta anónima para recabar la opinión y valoración de los estudiantes de la experiencia con las lecciones swirl con una tasa de respuesta de $1 / 3$ de los matriculados. Si consideramos que aproximadamente la mitad de los estudiantes se involucraron en la actividad y, por tanto, serían los candidatos a rellenar la encuesta, la tasa de respuesta estaría en torno a los $2 / 3$. Puntuando entre 0 y 10 , la nota media que le asignan al contenido de las lecciones es 8.1, sin embargo, la valoración de su experiencia baja al 6.8. Esto sugiere que debemos realizar alguna corrección en el diseño usado, algunos estudiantes las consideran largas y repetitivas, además de comentar que necesitan más ayuda para corregir las respuestas incorrectas.

En la Tabla 2 se recogen una serie de afirmaciones que se plantearon en la encuesta y el porcentaje de alumnos que está de acuerdo con ellas. De esta tabla se desprende que un alto porcentaje de encuestados considera estas lecciones útiles para entender, aprender y repasar la asignatura (afirmaciones 1, 2, 3, 4 y 5). Otros estudios con resultados similares son Henly (2003), Kibble (2007), Dinov, Sanchez y Christou (2008) y O'Dwyer, Carey y Kleiman (2007). Por otro lado, alrededor del $40 \%$ afirman que no trabajarían la asignatura a lo largo del cuatrimestre si no tuvieran que hacer estas lecciones o los exámenes intermedios (afirmaciones 8, 9 y 10). En torno a la mitad de los estudiantes les gustaría que en otras asignaturas también hubiera lecciones de este tipo, donde casi la mitad de ellos las habían considerado un trabajo excesivo.

Tabla 2 Porcentaje de estudiantes, ordenados de mayor a menor, que están de acuerdo con las afirmaciones.

\begin{tabular}{|c|c|}
\hline $\begin{array}{l}\text { Afirmación } \\
\end{array}$ & Estudiantes \\
\hline $\begin{array}{l}\text { 1. Las lecciones swirl me sirven para repasar la } \\
\text { asignatura a lo largo del cuatrimestre. }\end{array}$ & $90 \%$ \\
\hline $\begin{array}{l}\text { 2. Las lecciones swirl me ayudan a "entender" el } \\
\text { contenido de la asignatura. }\end{array}$ & $84 \%$ \\
\hline $\begin{array}{l}\text { 3. Las lecciones swirl me ayudan a "aprender" la } \\
\text { asignatura. }\end{array}$ & $84 \%$ \\
\hline 4. Las lecciones swirl son muy útiles. & $77 \%$ \\
\hline $\begin{array}{l}\text { 5. Creo que gracias a las lecciones swirl voy a } \\
\text { aprender más y a mejorar la nota de los exámenes } \\
\text { de la asignatura. }\end{array}$ & $68 \%$ \\
\hline $\begin{array}{l}\text { 6. La realización de las lecciones swirl suponen una } \\
\text { carga de trabajo excesiva. }\end{array}$ & $52 \%$ \\
\hline $\begin{array}{l}\text { 7. Me gustaría que en otras asignaturas también } \\
\text { hubiera lecciones de este tipo. }\end{array}$ & $48 \%$ \\
\hline $\begin{array}{l}\text { 8. Si no fuera por las lecciones swirl no trabajaría la } \\
\text { asignatura a lo largo del cuatrimestre. }\end{array}$ & $42 \%$ \\
\hline $\begin{array}{l}\text { 9. Hago las lecciones swirl solo porque tienen bonus } \\
\text { sobre la nota. }\end{array}$ & $42 \%$ \\
\hline 10. Solo estudio cuando tengo algún examen. & $39 \%$ \\
\hline
\end{tabular}

\section{B. Resultados académicos}

Los resultados académicos analizados corresponden a las notas obtenidas en las pruebas antes de sumar el bonus. Estudiaremos y compararemos las notas de cinco cursos académicos, desde el 2014-15 hasta el 2018-19, donde solo en este último curso tuvieron la opción de realizar lecciones swirl. Además, como no todos los alumnos eligieron realizarlas, compararemos también estos dos grupos de alumnos. Para comparar las distribuciones de notas estudiaremos el coeficiente de asimetría, la nota media e indicadores como la tasa de evaluación (TEV, presentados sobre matriculados), la tasa de éxito (TEX, aprobados sobre presentados) y la tasa de rendimiento (TR, aprobados sobre matriculados), donde $\mathrm{TR}=\mathrm{TEX} \cdot \mathrm{TEV}$. 
Si comparamos el grupo de estudiantes que optó por la realización de estas lecciones, realizándolas todas, con el grupo que no optó (hace 0,1 o 2) se puede observar un impacto positivo en las notas. Respecto a la prueba 1, la realización de las lecciones parece producir un desplazamiento de las notas hacia la derecha (Figura 2) y una modificación de la asimetría hacia la asimetría a la izquierda que es estadísticamente significativa con el test de Randles (Tabla 3). Esta misma pauta también parece observarse en la prueba 2 (Figura 3 ) aunque con menos intensidad, no siendo significativa (Tabla 3). La mejoría de las notas también queda reflejada en el incremento generalizado de todas las medidas de posición calculadas en ambas pruebas (Figura 4).

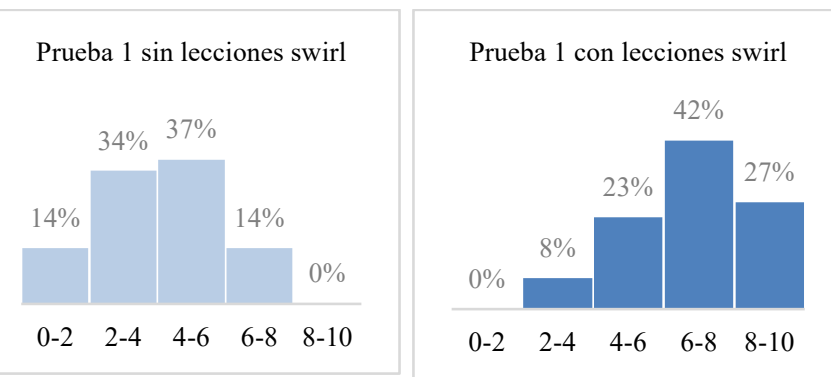

Figura 2 Distribución de las notas de la prueba 1 con y $\sin$ la realización de lecciones swirl en el curso 2018-2019.
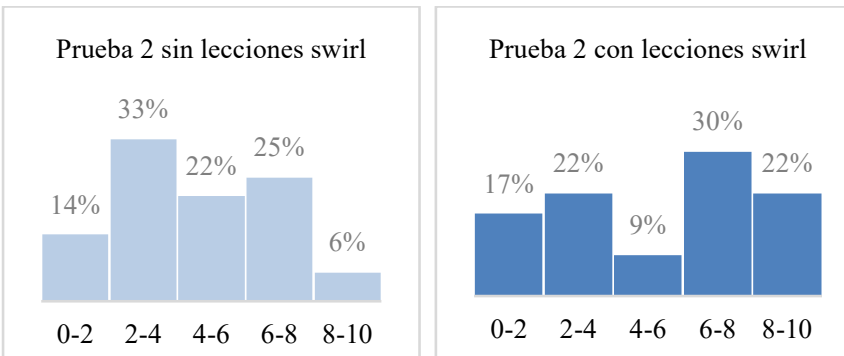

Figura 3 Notas de la prueba 2 con y sin la realización de lecciones swirl en el curso 2018-2019.

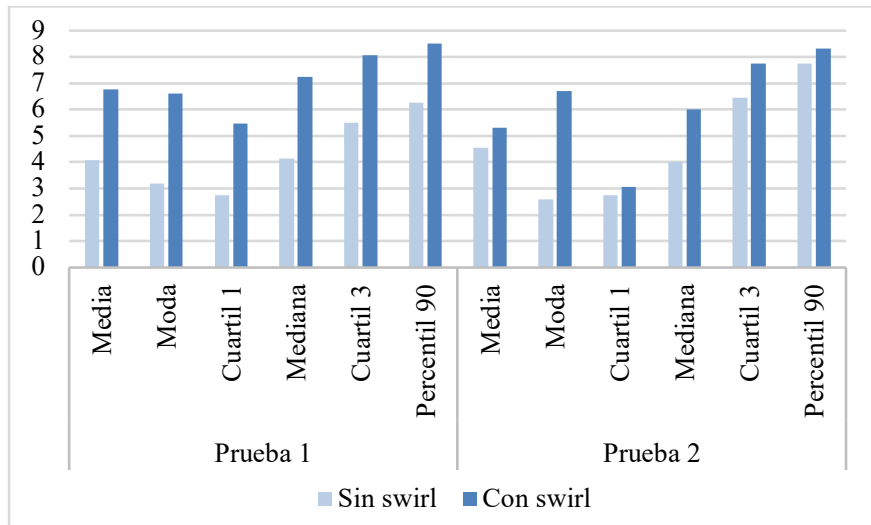

Figura 4 Medidas de posición de las notas de las pruebas 1 y 2 con y sin la realización de lecciones swirl en el curso 2018-2019.

De acuerdo con los resultados en la Tabla 3, los estudiantes que realizan las lecciones swirl obtienen mejor nota media en ambas pruebas, siendo la diferencia de medias muy alta y estadísticamente significativa en la prueba 1 usando el test de Welch ( $\mathrm{p}$-valor $<0.01)$. La tasa de éxito también experimenta un crecimiento importante en ambas pruebas, siendo de casi 50 puntos porcentuales en la prueba 1 y de 15.3 en la prueba 2 , aunque solo resulta estadísticamente significativo en la primera. En la prueba 1, teniendo una tasa de evaluación similar, la tasa de rendimiento es significativamente mayor cuando se han realizado las lecciones. Esto es, el porcentaje de estudiantes matriculados que aprueban es significativamente mayor entre los que han hecho las lecciones. Sin embargo, la tasa de evaluación es bastante diferente en la prueba 2 al disminuir el número de estudiantes que continúan haciendo lecciones. De ahí que no sea aconsejable comparar la tasa de rendimiento en la prueba 2 .

Tabla 3 Comparación del grupo de estudiantes que realizó las lecciones swirl con el grupo que no las realizó durante el curso 2018-2019.

\begin{tabular}{lccc}
\hline & \multicolumn{3}{c}{ Prueba 1 } \\
\cline { 2 - 4 } TEV & Sin swirl & Con swirl & p-valor \\
\cline { 2 - 4 } Asimetría & $30.10 \%$ & $28.00 \%$ \\
Nota media & $0.1(0.8176)$ & $-0.57(0.0622 *)$ & \\
TEX & 4.1 & 6.8 & $\left(0.0000^{* * *}\right)$ \\
TR & $35.70 \%$ & $84.60 \%$ & $\left(0.0007^{* * *}\right)$ \\
\hline & $10.80 \%$ & $23.70 \%$ & $\left(0.0326^{* *}\right)$ \\
\cline { 2 - 4 } & \multicolumn{3}{c}{ Prueba 2} \\
TEV & Sin swirl & Con swirl & p-valor \\
Asimetría & $0.31(0.1363)$ & $24.70 \%$ \\
Nota media & 4.5 & $-0.3(0.4366)$ \\
TEX & $41.20 \%$ & 5.3 \\
TR & $22.60 \%$ & $56.50 \%$ & $(0.2564)$ \\
* Diferencia significativa (p-valor $<0.1)$. & $(0.3301)$ \\
** Diferencia significativa (p-valor $<0.05)$. & \\
*** Diferencia significativa (p-valor $<0.01)$. &
\end{tabular}

Cuando se comparan los resultados del curso 2018-19 con los de cursos anteriores se obtienen resultados cualitativamente similares. En los últimos dos cursos los estudiantes dispusieron de un manual o texto-guía que también parece colaborar en la mejoría de los resultados académicos. En la Figura 5 se puede observar como todas las medidas de posición calculadas son más altas en el curso 2018-19, poniendo en evidencia un desplazamiento de la distribución de las notas cuando se usan las lecciones swirl. Esto sugiere que estas lecciones pueden beneficiar a la mayoría de los estudiantes y no solo a los mejores.

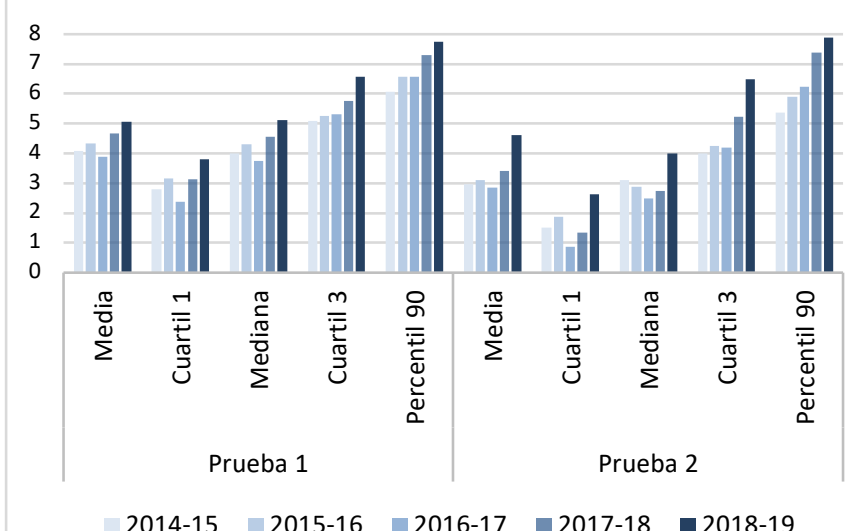

Figura 5 Evolución de las medidas de posición de las notas obtenidas en las pruebas 1 y 2 . 
La nota media de la prueba 1 del curso 2018-19 es mayor a la del curso anterior, pero el incremento no es estadísticamente significativo (Tabla 4). Sin embargo, si se compara con la de otros cursos sí resulta significativamente mayor. Respecto a la prueba 2, su nota media resulta significativamente mayor comparada con cualquiera de los cursos anteriores. La asimetría de las notas en la prueba 1 tiende a ser positiva excepto cuando se usan las lecciones swirl en el curso 2018-19, aunque el test de Randles no encuentra evidencia a favor de rechazar la simetría de los datos excepto para el curso 2017-18. En cambio, para la prueba 2 sí se puede rechazar la hipótesis de distribución simétrica, siendo la asimetría positiva, excepto en el último curso donde se usaron las lecciones swirl. Esto es, la introducción de las lecciones swirl parece combatir la asimetría a la derecha de las notas observada en los cursos anteriores.

Tabla 4 Resultados estadísticos de las pruebas 1 y 2 desde el curso 2014-15 al 2018-19.

\begin{tabular}{|c|c|c|c|c|c|}
\hline & 2014-15 & $2015-16$ & $2016-17$ & $2017-18$ & $2018-19$ \\
\hline Alumnos & 91 & 105 & 99 & 87 & 93 \\
\hline \multicolumn{6}{|c|}{ Prueba 1} \\
\hline Eventos & & & & Texto-guía & $\begin{array}{l}\text { Texto-guía } \\
\text { Lecc. swirl }\end{array}$ \\
\hline $\begin{array}{l}\text { TEV } \\
\text { p-valor1 }\end{array}$ & $91.2 \%$ & $\begin{array}{c}90.5 \% \\
(1.0000)\end{array}$ & $\begin{array}{c}96.0 \% \\
(0.2037)\end{array}$ & $\begin{array}{c}89.7 \% \\
(0.1632)\end{array}$ & $\begin{array}{c}97.8 \% \\
\left(0.0475^{* *}\right)\end{array}$ \\
\hline $\begin{array}{l}\text { Asim. } \\
\text { p-valor }\end{array}$ & $\begin{array}{c}0.5 \\
(0.3080)\end{array}$ & $\begin{array}{c}0.3 \\
(0.2996)\end{array}$ & $\begin{array}{c}0.2 \\
(0.2586)\end{array}$ & $\begin{array}{c}0.5 \\
(0.0724 *)\end{array}$ & $\begin{array}{r}-0.3 \\
(0.2651)\end{array}$ \\
\hline $\begin{array}{l}\text { Media } \\
{\text { p-valor } 1^{1}}{ }^{\text {p-valor }}{ }^{2} \\
\text { p-valor } 3^{3}\end{array}$ & 4.1 & $\begin{array}{c}4.3 \\
(0.3139)\end{array}$ & $\begin{array}{c}3.9 \\
(0.1403) \\
(0.5734)\end{array}$ & $\begin{array}{l}4.7 \\
(0.0182 * *) \\
(0.2210) \\
\left(0.0006^{* *}\right)\end{array}$ & $\begin{array}{c}5.1 \\
(0.2001) \\
(0.0002 * * *) \\
\left(0.0070^{* * *}\right)\end{array}$ \\
\hline $\begin{array}{l}\text { TEX } \\
\text { p-valor1 } \\
\text { p-valor2 }\end{array}$ & $27.7 \%$ & $\begin{array}{c}34.7 \% \\
(0.3980)\end{array}$ & $\begin{array}{r}33.7 \% \\
(1.0000) \\
0.4559\end{array}$ & $\begin{array}{r}44.9 \% \\
(0.1789) \\
(0.2295)\end{array}$ & $\begin{array}{c}56.0 \% \\
(0.1957) \\
(0.0042 * * *)\end{array}$ \\
\hline $\begin{array}{l}\text { TR } \\
\text { p-valor1 } \\
\text { p-valor2 }\end{array}$ & $25.3 \%$ & $\begin{array}{r}31.4 \% \\
(0.4280)\end{array}$ & $\begin{array}{c}32.3 \% \\
(1.0000) \\
(0.2733)\end{array}$ & $\begin{array}{r}40.2 \% \\
(0.3332) \\
(0.2637)\end{array}$ & $\begin{array}{c}54.8 \% \\
(0.0701 *) \\
(0.0027 * * *)\end{array}$ \\
\hline \multirow[b]{2}{*}{ Eventos } & \multicolumn{5}{|c|}{ Prueba 2} \\
\hline & & & Texto-guía & Texto-guía & $\begin{array}{l}\text { Texto-guía } \\
\text { Lecc. swirl }\end{array}$ \\
\hline $\begin{array}{l}\text { TEV } \\
\text { p-valor1 }\end{array}$ & $91.2 \%$ & $\begin{array}{c}87.6 \% \\
(0.5627)\end{array}$ & $\begin{array}{c}96.0 \% \\
(0.0573 *)\end{array}$ & $\begin{array}{c}80.5 \% \\
\left(0.0019^{* * *}\right)\end{array}$ & $\begin{array}{c}87.1 \% \\
(0.3137)\end{array}$ \\
\hline $\begin{array}{l}\text { Asim. } \\
\text { p-valor }\end{array}$ & $\begin{array}{c}0.4 \\
\left(0.0817^{*}\right)\end{array}$ & $\begin{array}{c}0.6 \\
\left(0.0100^{* * *}\right)\end{array}$ & $\begin{array}{c}0.7 \\
\left(0.0005^{* * *}\right)\end{array}$ & $\begin{array}{c}0.8 \\
\left(0.0000^{* * *}\right)\end{array}$ & $\begin{array}{c}0.2 \\
(0.1770)\end{array}$ \\
\hline $\begin{array}{l}\text { Media } \\
\text { p-valor1 } \\
\text { p-valor2 }\end{array}$ & 3.0 & $\begin{array}{c}3.1 \\
(0.6033)\end{array}$ & $\begin{array}{c}2.9 \\
(0.5123) \\
(0.4248)\end{array}$ & $\begin{array}{c}3.4 \\
(0.2011) \\
(0.4248)\end{array}$ & $\begin{array}{c}4.6 \\
\left(0.0044^{* * *}\right) \\
\left(0.0000^{* * *}\right)\end{array}$ \\
\hline $\begin{array}{l}\text { TEX } \\
\text { p-valor1 } \\
\text { p-valor2 }\end{array}$ & $13.3 \%$ & $\begin{array}{c}18.5 \% \\
(0.4623)\end{array}$ & $\begin{array}{c}22.1 \% \\
(0.6639) \\
(0.1498)\end{array}$ & $\begin{array}{c}25.7 \% \\
(0.7234) \\
(0.3597)\end{array}$ & $\begin{array}{c}43.2 \% \\
\left(0.0380^{* *}\right) \\
\left(0.0046^{* * *}\right)\end{array}$ \\
\hline $\begin{array}{l}\text { TR } \\
\text { p-valor1 } \\
\text { p-valor2 }\end{array}$ & $12.1 \%$ & $\begin{array}{c}16.2 \% \\
(0.5393)\end{array}$ & $\begin{array}{c}21.2 \% \\
(0.4588) \\
(0.1063)\end{array}$ & $\begin{array}{c}20.7 \% \\
(1.0000) \\
(0.5379)\end{array}$ & $\begin{array}{c}37.6 \% \\
(0.0199 * *) \\
(0.0191 * *)\end{array}$ \\
\hline
\end{tabular}

${ }^{1} \mathrm{p}$-valor1 se refiere a la diferencia respecto al curso anterior.

${ }^{2} \mathrm{p}$-valor2 se refiere a la diferencia respecto a 2 cursos antes.

${ }^{3} \mathrm{p}$-valor 3 se refiere a la diferencia respecto a 3 cursos antes.

El porcentaje de alumnos que decide presentarse a una prueba, dado por la tasa de evaluación, parece mostrar un comportamiento errático a lo largo de los distintos cursos, tanto en la prueba 1 como en la prueba 2 . No parece que pueda ser explicable por los eventos o hitos que se han producido en la asignatura. Sin embargo, tanto la tasa de éxito como la tasa de rendimiento sí que podrían verse afectadas al tener en cuenta los alumnos que aprueban. La tasa de éxito de la prueba 1 es significativamente mayor en el curso 2018-19 respecto a los cursos donde no había ni texto-guía ni lecciones swirl. Sin embargo, la prueba 2 tiene una nota media, tasa de éxito y tasa de rendimiento significativamente mayor comparada con cualquiera de los cursos anteriores. Esto es, el uso de las lecciones swirl junto con el texto guía ha tenido un impacto directo y positivo sobre los resultados de la prueba 2. Parece que el texto-guía no fue suficiente para lograr un incremento significativo de la nota media de esta prueba, lo que podría ser explicado por su mayor nivel de dificultad reflejado habitualmente por una nota media baja. El texto-guía por sí solo, sin embargo, parece que ha supuesto un cambio en los resultados de la prueba 1 incrementando su nota media y tasa de éxito, siendo el primero estadísticamente significativo. Respecto al porcentaje de los alumnos matriculados que aprueban (tasa de rendimiento), se observa un incremento significativo en las dos pruebas en el curso donde se usaron las lecciones swirl.

\section{Transferibilidad}

Las lecciones diseñadas tienen unas características básicas que hacen que el modelo sea transferible a otras disciplinas y otras herramientas. Esas características, junto con el nivel de implicación del estudiante, pueden ser en buena medida responsables de sus efectos positivos sobre el aprendizaje. En particular, podemos destacar las siguientes:

1. Cubren una gran parte del temario.

2. Alternan teoría (explicaciones, interpretaciones de resultados, etc.) y práctica.

3. Se desarrollan secuencialmente sin posibilidad de saltar ninguna pregunta o elegir qué hacer.

4. No se puede avanzar hasta que no se dé la respuesta correcta a una pregunta.

5. Ofrecen ayuda cuando se falla al contestar.

6. Se obtiene una nota que penaliza los intentos de respuesta fallidos, aunque cuando se llega al final se ha contestado todo correctamente.

7. Se pueden repetir ofreciendo datos, gráficos o imágenes diferentes en cada repetición.

8. Se han pautado temporalmente con entregas semanales.

9. El tiempo de duración depende de la destreza del estudiante, pero hay que procurar que no sean largas (unos 3045 minutos máximo).

10. El estudiante generalmente no percibe los beneficios de estas lecciones por lo que se debe incentivar su realización.

\section{CONCLUSIONES}

El uso de las lecciones interactivas swirl de $\mathrm{R}$ tiene grandes beneficios que, desde nuestro punto de vista, serían extensibles a cualquier material con una metodología similar. Los estudiantes disponen, además, de un texto-guía elaborado específicamente para la asignatura. Con la realización de estas lecciones se obtiene una mejoría en los resultados académicos que puede observarse tanto con respecto a otros años en los que no se hacían, como dentro del mismo curso académico al comparar los estudiantes que las hicieron frente a los que no. Comparando con otros cursos académicos, cuando se utilizan estas lecciones para preparar un examen se observa un 
incremento de la nota media, la tasa de éxito y la tasa de rendimiento que es estadísticamente significativo cuando la prueba tiene mayor dificultad. En ese caso la nota media creció un $35 \%$, el porcentaje de aprobados se incrementó en un $68 \%$ y el porcentaje de aprobados sobre matriculados en un $82 \%$. Cuando el nivel de dificultad es bajo, la introducción del textoguía parece suficiente para mejorar de forma significativa los resultados globales, aunque siguen mejorando con la introducción de estas lecciones. En este caso la nota media creció un $8.5 \%$, el porcentaje de aprobados un $25 \%$ y el de aprobados sobre matriculados un $36 \%$. Dentro del mismo curso académico se observa que la prueba 1 la aprobaron el $84.6 \%$ de los que realizaron las lecciones swirl con una nota media de 6.8 frente al $35.7 \%$ de los que no las realizaron con una nota media de 4.1. Para la prueba 2 estos porcentajes han sido, respectivamente, $56.5 \%$ (nota media 5.3 ) y $41.2 \%$ (nota media 4.5).

Para conseguir que el estudiante se involucre en esta actividad mejorada por la tecnología y obtenga sus beneficios, parece aconsejable que la actividad reúna ciertas características. Entre ellas, dar un incentivo para que las hagan, pautar temporalmente su realización para que el ritmo sea el adecuado, penalizar los intentos fallidos para que quieran repetirlas, hacer un recorrido por toda la asignatura para que repasen todo el programa, calibrar el contenido para que no sea un trabajo excesivo, y utilizar datos aleatorios para que cada estudiante se centre en su actividad y las repeticiones tengan algún elemento nuevo.

\section{REFERENCIAS}

Angus, S. D., \& Watson, J. (2009). Does regular online testing enhance student learning in the numerical sciences? Robust evidence from a large data set. British Journal of Educational Technology, 40(2), 255-272. https://doi.org/10.1111/j.1467-8535.2008.00916.x

Beetham, H., \& Sharpe, R. (2013). Rethinking Pedagogy for a Digital Age: Designing for 21st century learning (Edición: 2). New York: Routledge.

Dinov, I. D., Sanchez, J., \& Christou, N. (2008). Pedagogical utilization and assessment of the statistic online computational resource in introductory probability and statistics courses. Computers \& Education, 50(1), 284-300.

https://doi.org/10.1016/j.compedu.2006.06.003

Dunn, T. J., \& Kennedy, M. (2019). Technology Enhanced Learning in higher education; motivations, engagement and academic achievement. Computers \& Education, 137, 104-113. https://doi.org/10.1016/j. compedu.2019.04.004

Fonseca, D., Martí, N., Redondo, E., Navarro, I., \& Sánchez, A. (2014). Relationship between student profile, tool use, participation, and academic performance with the use of Augmented Reality technology for visualized architecture models. Computers in Human Behavior, 31, 434-445. https://doi.org/10.1016/j.chb.2013.03. 006

Fredricks, J. A., Blumenfeld, P. C., \& Paris, A. H. (2004). School Engagement: Potential of the Concept, State of the Evidence. Review of Educational Research, 74(1), 59-109. https://doi.org/10.3102/00346543074001059

Henly, D. C. (2003). Use of Web-based formative assessment to support student learning in a metabolism/nutrition unit. European Journal of Dental Education, 7(3), 116-122. https://doi.org/10.1034/j.1600-0579.2003. 00310.x

Huang, C. S. J., Su, A. Y. S., Yang, S. J. H., \& Liou, H.-H. (2017). A collaborative digital pen learning approach to improving students' learning achievement and motivation in mathematics courses. Computers \& Education, 107, 31-44. https://doi.org/10.1016/j. compedu.2016.12.014

Kahu, E. R. (2013). Framing student engagement in higher education. Studies in Higher Education, 38(5), 758773. https://doi.org/10.1080/03075079.2011.598505

Kennedy, M., \& Dunn, T. (2018). Improving the Use of Technology Enhanced Learning Environments in Higher Education in the UK: A Qualitative Visualization of Students' Views. 9(1), 76-89.

Kibble, J. (2007). Use of unsupervised online quizzes as formative assessment in a medical physiology course: effects of incentives on student participation and performance. Advances in Physiology Education, 31(3), 253-260. https://doi.org/10.1152/advan.00027. 2007

Lin, S. S. J., Liu, E. Z. F., \& Yuan, S. M. (2001). Web-based peer assessment: feedback for students with various thinking-styles. Journal of Computer Assisted Learning, 17(4), 420-432. https://doi.org/10.1046/j. 0266-4909.2001.00198.x

Margaryan, A., Littlejohn, A., \& Vojt, G. (2011). Are digital natives a myth or reality? University students' use of digital technologies. Computers \& Education, 56(2), 429-440.

McSweeney, L., \& Weiss, J. (2003). Assessing the math online tool: A progress report. Mathematics and Computer Education, 37(3), 248-267.

O’Dwyer, L. M., Carey, R., \& Kleiman, G. (2007). A Study of the Effectiveness of the Louisiana Algebra I Online Course. Journal of Research on Technology in Education, 39(3), 289-306. https://doi.org/10.1080/ 15391523.2007 .10782484

Universities and Colleges Information Systems Association. (2016). 2016 Survey of Technology Enhanced Learning for higher education in the UK.

Wang, M.-T., \& Eccles, J. S. (2012). Adolescent Behavioral, Emotional, and Cognitive Engagement Trajectories in School and Their Differential Relations to Educational Success. Journal of Research on Adolescence, 22(1), 31-39. https://doi.org/10.1111/j.1532-7795.2011. 00753. $\mathrm{x}$

Whitehead, J. (1984). Motives for higher education: a study of intrinsic and extrinsic motivation in relation to academic attainment. Cambridge Journal of Education, 14(2), 26-34. https://doi.org/10.1080/ 0305764840140204 